\title{
FOCUSING OF ALFVÉNIC POWER IN NEUTRON STAR MAGNETOSPHERES
}

\author{
Marco Fatuzzo * \\ Dept. of Physics, University of Michigan, Ann Arbor, MI 48109 \\ Fulvio Melia ** \\ Dept. of Physics and Steward Observatory, U. of Arizona, Tucson, AZ 85721
}

\begin{abstract}
Highly dynamic magnetospheric perturbations in neutron star environments can naturally account for the features observed in Gamma-ray Burst spectra. However, if GRB's have an extragalactic origin, as is implied by the uniform yet spatially truncated distribution observed by the BATSE experiment, then noncatastrophic isotropic emission mechanisms may be ruled out on energetic and timing arguments. As such, we consider MHD processes which can produce strongly anisotropic $\gamma$-rays with an observable flux out to distances of 1-2 Gpc. In particular, we show that sheared Alfvén waves propagating along open magnetospheric field lines at the poles of magnetized neutron stars transfer their energy dissipationally to the charges generating the current which sustains the field misalignment, and thereby focus their power into a spatial region (i.e., the shear) that can be many times smaller than that of the crustal disturbance. This produces a strong (observable) flux enhancement along certain directions.
\end{abstract}

\section{INTRODUCTION}

The lack of a precise determination of a distance scale to Gamma-ray burst (GRB) sources has greatly hindered our theoretical understanding of these objects. Much of what we know about these bursts is based on inferences drawn from clues provided by their spectra, including their rapid variability, their complex temporal structure, and the emission of a substantial fraction of their power at energies in excess of $\sim 1 \mathrm{MeV}$.

While it is possible to account for these observations by invoking a model in which the bursts originate within the magnetosphere of strongly magnetized neutron stars, this paradigm must be reconciled with the uniform, yet spatially truncated GRB distribution observed by the BATSE experiment on CGRO ${ }^{1}$. These observations seem to rule out nearby (i.e. Galactic) single population models, and have therefore led to renewed speculation that GRB's originate at cosmological redshifts. But a naive estimate of the burst energy required for such distant sources yields a value that is significantly larger than that which a neutron star could reasonably produce unless the event was catastrophic (e.g., the coalescence of a neutron-star binary ${ }^{2}$ ), which does not seem to be borne out by the time history of typical bursts.

A resolution to this apparent conflict was proposed by Melia and Fatuzzo ${ }^{3}$, in which sheared Alfvén waves generated near the polar cap of strongly magnetized neutron stars produce streams of relativistic particles that are focused by

* Compton GRO Fellow

** Alfred P. Sloan Fellow 


\section{Focusing of Alfvénic Power in Neutron}

the underlying magnetospheric structure. These energetic charges upscatter the radio-frequency photons (emitted at larger radii) into $\gamma^{-1}$ cones aligned with the underlying magnetic field lines, resulting in an enhanced $\gamma$-ray flux along preferred lines of sight. This anisotropic emission is such that a pulsar glitch releasing $\sim 10^{45}$ ergs of energy could be viewed as a GRB out to a distance of $\gtrsim 1$ Gpc. A key assumption of this scenario is that the Alfvénic power can indeed be emitted anisotropically. We show here that the required focusing is a natural consequence of the dissipational properties of sheared Alfvén waves whose shear lengthscales $(s \leqslant 10 \mathrm{~cm})$ are much smaller than the size of the region $\left(Z 10^{4}\right.$ $\mathrm{cm})$ encompassing the overall Alfvén wave fluctuation.

\section{SHEARED ALFVÉN WAVE DISSIPATION}

Sheared Alfvén waves may be described by magnetic perturbations of the form

$$
\mathbf{B}_{\mathrm{A}}=B_{\mathbf{a}}(y) \exp (i k z-i \omega t) \hat{x},
$$

where $B_{\mathrm{a}}(y)$ is an odd function that characterizes the shear geometry. It is clear from the form of $B_{\mathrm{a}}(y)$ and Ampére's Law that an electric field $E_{\mathrm{Az}}$ must exist inside the sheared region until a sufficiently strong current $J_{\mathrm{s}}$ is produced parallel to the underlying magnetic field $\mathbf{B}_{0}=B_{0} \hat{z}$. If the equilibrium Goldreich-Julian particle density $n_{0}$ is insufficient to support a current large enough to short out $E_{\mathrm{Az}}$, charges must be copiously stripped off the stellar surface, thereby inducing a charged particle flow to give the required $J_{\mathrm{s}}$. Since the Alfvén speed in these environments is $v_{\alpha} \gg c$, the waves travel with a phase velocity $u_{\alpha}=\omega / k \approx c$. In order for $J_{s}$ and the encompassing magnetic shear to remain in phase, the particle flow must be relativistic, and thus, have an average density

$$
n_{\mathrm{s}} \approx \frac{B_{\mathrm{a} 0}}{4 \pi e s} \approx 10^{19} \mathrm{~cm}^{-3}\left(\frac{B_{\mathrm{a} 0}}{10^{12} \mathrm{G}}\right)\left(\frac{s}{10 \mathrm{~cm}}\right)^{-1}
$$

where $B_{\mathrm{a} 0}$ is the magnitude of the magnetic perturbation and $s$ is the lengthscale of the shear (which is assumed to be much smaller than the lengthscale $S$ of the encompassing plane wave regions). For convenience, we define $\eta \equiv S / s$. We note that the stripped particles escape from the system by flowing out along the open magnetospheric field lines.

In standard pulsar theory, radio emission results from the coherent motion of "bunches" of electrons streaming along open field lines with Lorentz factors $\gamma \sim 10^{4-5}$. As such, strong transient radio emission is expected to be a natural byproduct of sheared Alfvén waves if similar particle energies are reached, and if this emission is produced with front-back symmetry along the local field-line direction, a large fraction of the overall radio flux will naturally be funneled back onto the polar cap. Taking into account the coherent nature of the processes responsible for pulsar emission, we parametrize the flux impinging onto the stellar surface by $F_{\mathrm{r}}=\xi \eta^{-2}\left(n_{\mathrm{s}} / n_{\mathrm{C}}\right)^{2}\left(L_{\mathrm{C}} / \pi R_{\mathrm{pc}}^{2}\right)$, where $L_{\mathrm{C}}$ and $n_{\mathrm{C}}$ are the Crab pulsar luminosity and magnetospheric number density, respectively, and where $R_{\mathrm{pc}}$ is the radius of the open field line polar cap. Assuming a stellar radius of $R_{*}=10^{6} \mathrm{~cm}, R_{\mathrm{pc}}$ can be related to the pulsar period $P$ via $R_{\mathrm{pc}}=$ $1.4 \times 10^{4} \mathrm{~cm}(P / 1 \mathrm{~s})^{-1 / 2}$. With $L_{\mathrm{C}}=10^{32} \mathrm{ergs} \mathrm{s}^{-1}$ and $n_{\mathrm{C}}=10^{13} \mathrm{~cm}^{-3}$, this 
yields

$$
F_{\mathrm{r}} \approx 10^{30} \mathrm{ergs} \mathrm{cm}^{-2} \mathrm{~s}^{-1} \xi\left(\frac{\eta}{10^{3}}\right)^{-2}\left(\frac{B_{\mathrm{a} 0}}{10^{12} \mathrm{G}}\right)^{2}\left(\frac{s}{10 \mathrm{~cm}}\right)^{-2}\left(\frac{P}{5 \mathrm{~s}}\right),
$$

where $\eta^{-1}=(S / s)^{-1}$ is the sheared flow "filling factor". The parameter $\xi$ encompasses both geometric and emission uncertainties, and as such, is poorly known. We note that if $\xi$ becomes too small ( $\leqslant 0.1$ for the range of parameters considered here), the wave dissipation lengthscale due to field line annhilation becomes much larger than $R_{*}$, and the SAW mechanism becomes inefficient at producing $\gamma$-rays. However, since the Crab pulsar is itself very inefficient at converting spin-down energy into radio emission compared to typical pulsars, and since we have made the conservative assumption that the (coherent) radio flux scales as $\eta^{-2}$ (i.e., the square of the total number of particles), it is reasonable to assume that $\xi \gg 0.1$ (see also the discussion below).

The presence of $F_{\mathrm{r}}$ results in a radiative drag on the relativistic currentcarrying charges. By analogy with MHD phenomena, the current driving electric field $\left(\mathbf{E}=E_{\mathrm{A} z} \hat{z}\right)$ must be generated within the shear at the expense of the magnetic wave energy. However, the sheared waves are distinguished from pure MHD fluctuations for two important reasons. First, the charges which generate $J_{\mathbf{s}}$ are constrained to always move along the same $\mathbf{B}_{0}$ field lines, so that SAWs cannot easily change their initial structure. Second, the simple concept of Ohm's law is not valid for the relativistic flow inside the shear. Indeed, once the particles become relativistic, the current quickly decouples from the driving electric field, and since the radiative drag increases rapidly with $\gamma$ (the particle Lorentz factor), one might expect that a mildly relativistic flow will be favored by the system. Though $E_{\mathrm{Az}}$ depends on the microphysics of the shear (including all the annihilation processes, such as the tearing mode instability), its value may be estimated with a relatively simple argument under the assumption that the annihilation time scale within the shear is the shortest of the relevant time scales. The strength of the electric field is limited by the rate at which the oppositely-directed magnetic fluctuations are driven together by the large magnetic pressure gradients associated with SAWs. Since the Alfvénic field lines are strongly coupled to $B_{0}$ via flux freezing with the charged medium, this transfer of Alfvénic power into the shear is dictated by the diffusion rate within the resistive plasma in the region $|y|>s$, where the resistance is provided primarily by $e^{-} /$radio photon scatterings. As long as most of the wave energy is channeled into the shear before the waves break, we may equate the Alfvénic luminosity generated at the stellar surface with the power dissipated by the current as it converts magnetospheric energy into upscattered racliation. This yields an average electric field strength

$$
\begin{aligned}
E_{\mathrm{Az}} & \approx \min \left[5 \times 10^{11} \mathrm{sV} \mathrm{cm}^{-1}\left(\frac{B_{\mathrm{a} 0}}{10^{12} \mathrm{G}}\right),\right. \\
& \left.10^{10} \mathrm{sV} \mathrm{cm}^{-1} \xi\left(\frac{B_{\mathrm{a} 0}}{10^{12} \mathrm{G}}\right)^{3}\left(\frac{P}{5 \mathrm{~s}}\right)^{2}\left(\frac{B_{0}}{10^{12} \mathrm{G}}\right)^{-1}\left(\frac{S}{10^{4} \mathrm{~cm}}\right)^{-3}\right],
\end{aligned}
$$

where we have used $\eta=S / s$. The first expression on the RHS of eq. (4) represents the maximum attainable value of $E_{\mathrm{Az}}$ and occurs when the diffusion velocity approaches $\mathrm{c}$. 


\section{Focusing of Alfvénic Power in Neutron}

We assume a typical pulsar spectrum specified as a steep power law with (flux density) index $\mu$ above a break at frequency $\epsilon_{0} / h \approx 500 \mathrm{MHz}$. With $\gamma \gg 1$, a lab frame photon with energy $\epsilon$ will be blue-shifted to $\sim 2 \gamma \epsilon$ in the electron rest frame, which is well below the resonant energy $\epsilon_{B} \equiv\left(B_{0} / 44.14 \times 10^{12} \mathrm{G}\right) m_{e} c^{2}$, and its angle of propagation relative to the particle direction (and hence $\mathbf{B}_{0}$ ) is $\sim \gamma^{-1}$. As such, $\epsilon_{B} / \epsilon \approx 6 \times 10^{9}\left(B_{0} / 10^{12} \mathrm{G}\right)(\epsilon / h 500 \mathrm{Mhz})^{-1}<\gamma^{2}$, and $\sigma_{\mathrm{MC}} \approx 4 \sigma_{T}\left(\gamma \epsilon / \epsilon_{B}\right)^{2} \quad 4,5$. Balancing the accelerating force $e E_{\mathrm{Az}}$ by the radiative drag $\gamma^{2} F_{\mathrm{r}} \sigma_{\mathrm{MC}} / c$, one therefore obtains

$$
\begin{aligned}
\gamma_{\mathrm{s}} \approx 10^{6} f(\xi) & \left(\frac{B_{\mathrm{a} 0}}{10^{12} \mathrm{G}}\right)^{1 / 4}\left(\frac{B_{0}}{10^{12} \mathrm{G}}\right)^{1 / 4} \\
& \left(\frac{P}{5 \mathrm{~s}}\right)^{1 / 4}\left(\frac{S}{10^{4} \mathrm{~cm}}\right)^{-1 / 4}\left(\frac{\epsilon_{0}}{h 500 \mathrm{MHz}}\right)^{-1 / 2},
\end{aligned}
$$

where $f(\xi)=\min \left[1,\left(\xi / \xi_{0}\right)^{-1 / 4}\right]$ and the parameter $\xi_{0}$ is defined as the smallest value of $\xi$ for which the diffusion velocity equals c (e.g., $\xi_{0}=35$ for the represented parameter space). This result is consistent with the assumptions discussed above (e.g., that the particle motion is relativistic and sufficiently energetic to produce the required raclio luminosity and that $\sigma_{M C}$ have an $\epsilon^{2}$ dependence). Ultimately, the current $J_{\mathrm{s}}$ must decay in concert with the Alfvénic magnetic field, even though the particles remain relativistic. Evidently, the initially fully charge separated regions must merge together and neutralize. This behavior is expected since the power transferred from the wave to the particles is reduced as the magnetic field decays. As such, an increasing number of charges undergoing collisions will not be energized quickly enough to remain in phase with the wave, and are therefore swept up by the lagging oppositely charged wave region.

The scaling of the magnetic fields $B_{0}$ and $B_{\mathrm{a} 0}$ in the above equations was chosen for convenience and not to suggest that $B_{\mathrm{a} 0} \sim B_{0}$. We note, however, that the presence of significant recomnection in the nonlinear regime would have the desirable effect of enhancing the amnihilation rate within the shear (see the paragraph preceeding Eq. 4).

\section{DISCUSSION}

In applying the above cliscussion to the cosmological gamma-ray burst model, we must now generalize to a more realistic magnetospheric geometry in which the field lines are more or less radial close to the stellar surface. It is evident from $\$ 2$ that SAWs focus their energy into the internal current flow. Thus, as long as $\gamma^{-1} \ll s / R_{*}$, the flux is enhanced by a factor $\eta$ in certain directions, for which a source at a distance $D$ will have an observable $\gamma$-ray flux

$$
F_{\gamma} \approx 10^{-7} \mathrm{ergs} \mathrm{cm}^{-2} \mathrm{~s}^{-1}\left(\frac{\eta}{10^{3}}\right)\left(\frac{B_{\mathrm{a} 0}}{10^{12 \mathrm{G}}}\right)^{2}\left(\frac{D}{1 \mathrm{Gpc}}\right)^{-2}
$$

The observability of these bursts at cosmological distances imposes strict (but not unrealistic) conditions on the model parameters, such as the required burst power $\left(L_{\text {burst }} \sim 10^{44}-10^{46}\right.$ ergs s ${ }^{-1}$ ), whose magnitude depends on whether 
the SAWs are generated only near the polar cap (where the field lines are most strongly coupled to toroidal crustal activity) or are generated throughout the entire stellar surface.

Since the particle flow remains optically thin to the radio photons impinging upon the star, the $\epsilon^{2}$ dependence of the cross-section (see above) implies that the incipient radio spectrum is upscattered to a $\gamma$-ray spectrum with (power) index $\mu+2+1$, and very importantly, that the spectral radio break at $\epsilon_{0}$ is translated to the corresponding $\gamma$-ray break at

$$
\begin{aligned}
\epsilon_{\text {break }} \sim 2 \gamma_{\mathrm{s}}^{2} \epsilon_{0} \approx 4.4 \mathrm{MeV} g(\xi) \\
\left(\frac{B_{\mathrm{a} 0}}{10^{12} \mathrm{G}}\right)^{1 / 2}\left(\frac{B_{0}}{10^{12} \mathrm{G}}\right)^{1 / 2}\left(\frac{P}{5 \mathrm{~s}}\right)^{1 / 2}\left(\frac{S}{10^{4} \mathrm{~cm}}\right)^{-1 / 2},
\end{aligned}
$$

independent of $\epsilon_{0}$. Here, $g(\xi)=\min \left[1,\left(\xi / \xi_{0}\right)^{-1 / 2}\right]$. This result compares favorably with the observed value of $\epsilon_{\text {break }}$ (which after redshift is taken into account is seen to fall within the range $\sim 100 \mathrm{keV}-3 \mathrm{MeV}^{6}$ ), and suggests that $\xi / \xi_{0}$ may be as large as 100. A more detailed description of the resulting $\gamma$-ray spectrum is given in Reference 3 (see, for example, Figure 2 therein). For completeness, we note that a cylindrical shear would correspond to $\eta \sim(S / s)^{2}$ and the parameter $S$ in Equation (7) should be replaced by $s$. Such a strictly confined shear region would thus appear to be unlikely, though it cannot be ruled out without a more detailed calculation.

\section{REFERENCES}

1. C. A. Meegan et al., Nature 355, 143 (1992).

2. R. Narayan, B. Paczyński, and T. Piran, Ap. J. 395, L83 (1992).

3. F. Melia and M. Fatuzzo, Ap. J. 398, L85 (1992).

4. F. Melia and M. Fatuzzo, Ap. J. 346, 378 (1989).

5. C. D. Dermer, Ap. J. 360, 197 (1990).

6. B. E. Schaefer et al., Ap. J 393, L51 (1992). 